\title{
The geographic distribution and complex evolutionary history of the NX-2 trichothecene chemotype from Fusarium graminearum
}

Amy Kelly ${ }^{a}$, Robert H. Proctor ${ }^{a}$, Francois Belzile ${ }^{b}$, Sofia N. Chulze ${ }^{c}$, Randall M. Clear ${ }^{d}$, Christina Cowger ${ }^{\mathrm{e}}$, Wade Elmer ${ }^{\dagger}$, Theresa Lee ${ }^{g}$, Friday Obanor ${ }^{\mathrm{h}}$, Cees Waalwijk ${ }^{\mathrm{i}}$, and Todd J. Ward ${ }^{\mathrm{a}^{*}}$

${ }^{a}$ U.S. Department of Agriculture, Agricultural Research Service, 1815 North University Street, Peoria, IL 61604, USA; ${ }^{b}$ Université Laval, 1030 avenue de la Médecine, Québec City, Québec G1V 0A6, Canada; ' Universidad Nacional de Río Cuarto-CONICET.

Rutas 8 and 36 Km 601 (5800) Rio Cuarto, Córdoba, Argentina; d 23 Healy Crescent,

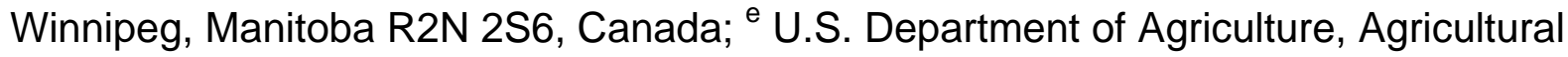
Research Service, 3411 Gardner Hall, North Carolina State University, Raleigh, NC 27695, USA; ${ }^{\dagger}$ The Connecticut Agricultural Experiment Station, 123 Huntington St, New Haven, CT 06504, USA; ${ }^{9}$ National Institute of Agricultural Sciences, 166 Nongsaengmyeong-ro, Iseo-myeon, Wanju-gun, Jeollabuk-do 55365, Republic of Korea; ${ }^{\mathrm{h}}$ Commonwealth Scientific and Industrial Research Organization, 306 Carmody Road, St Lucia QLD 4067, Australia; ' Plant Research International, Droevendaalsesteeg 1, 6708 PB Wageningen, The Netherlands

"Corresponding author. Address: U.S. Department of Agriculture, Agricultural Research Service, 1815 North University Street, Peoria, IL 61604, U.S. Phone: +1 3096816394. E-mail address: todd.ward@ars.usda.gov 


\section{Abstract}

Fusarium graminearum and 21 related species comprising the $F$. sambucinum species complex lineage 1 (FSAMSC-1) are the most important Fusarium Head Blight pathogens of cereal crops world-wide. FSAMSC-1 species typically produce type B trichothecenes. However, some F. graminearum strains were recently found to produce a novel type $\mathrm{A}$ trichothecene (NX-2) resulting from functional variation in the trichothecene biosynthetic enzyme Tri1. We used a PCR-RFLP assay targeting the TRI1 gene to identify the NX-2 allele among a global collection of 2,515 F. graminearum. NX-2 isolates were only found in southern Canada and the northern U.S., where they were observed at low frequency (1.8\%), but over a broader geographic range and set of cereal hosts than previously recognized. Phylogenetic analyses of TRI1 and adjacent genes produced gene trees that were incongruent with the history of species divergence within FSAMSC-1, indicating trans-species evolution of ancestral polymorphism. In addition, placement of NX-2 strains in the TRI1 gene tree was influenced by the accumulation of nonsynonymous substitutions associated with the evolution of the NX-2 chemotype, and a significant $(P<0.001)$ change in selection pressure was observed along the NX-2 branch $(\omega=1.16)$ in comparison to other branches $(\omega=0.17)$ in the TR/1 phylogeny. Parameter estimates were consistent with positive selection for specific amino-acid changes during the evolution of NX-2, but direct tests of positive selection were not significant. Phylogenetic analyses of four-fold degenerate sites and intron sequences in TR/1 indicated the NX-2 chemotype had a single evolutionary origin and evolved recently from a type B ancestor. Our results indicate the NX-2 chemotype may be indigenous, and possibly endemic, to southern 
Canada and the northern U.S. In addition, we demonstrate that the evolution of TRI1 within FSAMSC-1 has been complex, with evidence of trans-species evolution and chemotype-specific shifts in selective constraint.

\section{Keywords}

Fusarium head blight, trichothecene, NX-2, TR/1, phylogenetics, molecular evolution 


\section{Introduction}

Fusarium Head Blight (FHB) is a disease of wheat, barley and other cereal crops that can significantly reduce grain yield and quality, and can further diminish the value of grain due to contamination with trichothecenes and other mycotoxins (McMullen et al., 2012). Fusarium graminearum is the most common and economically important FHB pathogen world-wide (Del Ponte et al., 2015; Goswami and Kistler, 2004; Qu et al., 2008), and was responsible for billion dollar annual losses to the North American wheat industry in past epidemics (Nganje et al., 2004). F. graminearum and the other members of the F. graminearum species complex (FGSC) are included in a broader group of $21 \mathrm{FHB}$ pathogen species characterized by the production of type B trichothecene mycotoxins. This diverse group was previously referred to as the B-clade, but will be referred to here as Fusarium sambucinum species complex lineage 1 (FSAMSC-1) to reflect an exclusive evolutionary origin within the F. sambucinum species complex (O'Donnell et al., 2013) as opposed to an exclusive trichothecene type.

Trichothecene mycotoxins are prevalent grain contaminants that can represent a major threat to food safety and animal health. These toxins are potent inhibitors of protein synthesis and are responsible for neurologic, gastrointestinal, immune function and other disorders (Pestka, 2010; Wu et al., 2014). Trichothecenes can also act as virulence factors and facilitate tissue colonization in some plant hosts (Desjardins et al., 1996; Jansen et al., 2005; Mudge et al., 2006). Fusarium trichothecenes are divided into two major structural types characterized by the presence (type B) or absence (type A) of a keto group at carbon atom 8 (C-8). Members of the FSAMSC-1, for example, typically produce one of three strain-specific profiles (chemotypes) of 8-keto, 7-hydroxy, type B 
trichothecenes. These chemotypes are differentiated by the production of nivalenol (NIV), 3-acetyl deoxynivalenol (3ADON), or 15-acetyl deoxynivalenol (15ADON) (Miller et al., 1991; Ward et al., 2002). Recently however, a novel type A trichothecene chemotype (NX-2) was reported for several isolates of $F$. graminearum that produce 7hydroxy trichothecenes lacking a C-8 oxygen (Kelly et al., 2015; Liang et al., 2014; Varga et al., 2015) (Fig. 1). This structural variation at C-7 and C-8 results from functional diversity of the trichothecene biosynthetic enzyme Tri1, a cytochrome P450 monooxygenase. Strains of $F$. graminearum that produce NX-2 have a unique variant of Tri1 that catalyzes C-7 hydroxylation, but not C-8 hydroxylation (Varga et al., 2015). In contrast, Tri1 catalyzes C-7 and C-8 hydroxylation in type B trichothecene-producing members of FSAMSC-1, and catalyzes hydroxylation at C-8 but not C-7 in fusaria that produce C-8-oxygenated type A trichothecenes such as T-2 toxin, HT-2 toxin and/or neosolaniol (McCormick et al., 2004; McCormick et al., 2006; Meek et al., 2003). NX-2 toxicity appears similar to that of $3 A D O N$, and its deacetylated form (NX-3) is similar in toxicity to DON (Varga et al., 2015).

The evolution of trichothecene chemotype diversity within Fusarium has been complex. Some structural diversity among type B trichothecenes is due to genetic polymorphism in the trichothecene biosynthetic gene (TRI) cluster (Alexander et al., 2009; Lee et al., 2002) that has been maintained by balancing selection throughout the evolution of the FSAMSC-1 (Ward et al., 2002). In addition, trans-species evolution and multiple genomic translocations of the $T R / 1$ gene have been reported, such that $T R / 1$ is found in at least four genomic contexts (GC) and aspects of the TR/1 gene tree are incongruent with the species phylogeny (Proctor et al., 2009). In the B-trichothecene 
strains from FSAMSC-1 studied to date, TRI1 is in GC1; that is, between homologs of FGSG_00070 and FGSG_00072, and with a TR/16 pseudogene ( $\Psi T R / 16)$ in its 5' flanking region (Proctor et al., 2009). However, the genomic context and evolutionary affinities of the TR/1 variants from NX-2 isolates have not been investigated.

All NX-2 isolates identified to date were collected from southern Canada and the Upper Midwest of the U.S (Kelly et al., 2015; Liang et al., 2014). Two genetic populations of type B-producing F. graminearum have been described from these regions and consist of the native NA1 population in which the 15ADON chemotype predominates and the emergent NA2 population in which the 3ADON chemotype is predominant (Gale et al., 2007; Liang et al., 2015; Ward et al., 2008). However, the NX2-producing F. graminearum strains appear genetically distinct from the NA1 and NA2 genetic populations (Kelly et al., 2015; Liang et al., 2014). The presence of the NX-2 allele in $F$. graminearum populations outside of this region has not been investigated and the geographic distribution of NX-2 isolates across major cereal production regions of the world is not known. The objectives of this study were to 1) characterize the distribution of NX-2 isolates among a global collection of $F$. graminearum isolates, and 2) to elucidate the origins and evolutionary history of the NX-2 chemotype in $F$. graminearum.

\section{Methods}

\subsection{Geographic survey of NX-2 alleles in F. graminearum}

The isolates used in this study are described in Supplementary Table 1. Fungal cultures were grown and DNA was extracted following methods described by O'Donnell et al., (1998). A TR/1 PCR-RFLP assay (Liang et al., 2014) was used to screen $F$. 
graminearum isolates for the NX-2 TRI1 allele. Briefly, this assay targets TRI1 Apol restriction sites that are unique to NX-2-producing F. graminearum strains, such that the NX-2 TR/1 genotype can be distinguished from type B genotypes (i.e. 15ADON, 3ADON and NIV) of $F$. graminearum based on the number and length of restriction fragments. The PCR-RFLP assay was performed on a global collection of 2,515 F. graminearum, which included 1,170 isolates from previous studies of FHB composition and F. graminearum population genetics (Aamot et al., 2015; Bec et al., 2015; Boutigny et al., 2014; Boutigny et al., 2011; Del Ponte et al., 2015; Gale et al., 2007; Gale et al., 2011; Kuhnem et al., 2015; Reynoso et al., 2013; Suga et al., 2008; Umpiérrez-Failache et al., 2013; Ward et al., 2008; Yli-Mattila et al., 2009) and 1,345 additional isolates identified as F. graminearum using a multi-locus genotyping (MLGT) assay (Ward et al., 2008), with modifications described in Kelly et al., (2015).

\subsection{DNA sequencing and phylogenetic analyses}

We sequenced portions of $\beta$-tubulin (TUB2), translation elongation factor $1 \alpha$ (TEF1), trichothecene-3-O-acetyltransferase (TRI101), phosphate permease (PHO5), histone H3 (HIS3) and reductase (RED) from NX-2 isolates in order to integrate NX-2 diversity into the previously published phylogenetic framework for FSAMSC-1 (Sarver et al., 2011). In addition, we sequenced TR/1 from all isolates identified as NX-2, a subset of 239 geographically diverse $F$. graminearum isolates identified as type B based on the PCR-RFLP assay, and a previously characterized collection of isolates representing FSAMSC-1 diversity (Sarver et al. 2011) in order to validate RFLP results and to assess the evolutionary history of the TR/1 gene. We also sequenced a 5-kb region upstream and a 1.3-kb region downstream of the TR/1 locus in NX-2 isolates and B trichothecene 
isolates representing FSAMSC-1 diversity (Sarver et al., 2011) in order to assess genomic context and infer phylogenetic relationships based on variation in genes immediately flanking $T R I 1$. Sequencing of individual genes via capillary electrophoresis (Accessions KX087123-KX0871, KX183208-KX183387, KX183571-KX183628, KX183629-KX183685) was conducted using an ABI 3730 DNA Analyzer as previously described (Sarver et al., 2011; Varga et al., 2015). Primers used to amplify TRI1 and adjacent genes are provided in Supplementary Table 2. Additional TR/1 sequences were extracted from unreleased Illumina MiSeq (Illumina Inc., San Diego, California) whole-genome sequences of $F$. graminearum isolates (Accessions $\mathrm{KX} 183388$ KX183570) mapped to $~ 50 \mathrm{X}$ coverage against the F. graminearum $\mathrm{PH}-1$ reference genome (Accession PRJEB5475). Genome sequencing libraries were prepared using the Illumina Nextera XT DNA Library Prep Kit, following manufacturer's instructions for 2x300bp MiSeq runs. Amplified DNA sequences were trimmed to remove low-quality ends and overlapping amplicons were assembled into contigs using Sequencher 4.10.1 (Gene Codes Corporation, 2010). Trimming and assembly of genome reads was performed using CLC Genomics Workbench 8.0.1 (QIAGEN Bioinformatics, 2015). DNA sequences were aligned with MUSCLE (Edgar, 2004) as implemented in MEGA version 6.6 (Tamura et al., 2013). MEGA was used to construct maximum likelihood phylogenies, with models of molecular evolution selected on the basis of Bayesian Information Criterion scores. Support for individual nodes was assessed through random resampling of sequences with 1000 bootstraps. Alternate phylogenetic hypotheses were tested by comparing the fit of constrained versus unconstrained maximum likelihood phylogenies via Shimodaira-Hasegawa likelihood ratio tests (SH 
tests) (Shimodaira and Hasegawa, 1999) as implemented in RAxML version 8.0.26 (Stamatakis, 2014).

\subsection{Molecular evolution of NX-2 TRI1 alleles}

The ratio of nonsynonymous to synonymous substitution rates $(\omega)$ was determined for $T R / 1$ sequences to characterize the influence of different selection pressures on TR/1 evolution. We used the CODEML program in PAML (version 4.8, Yang, 2007) to obtain parameter estimates by maximizing the likelihood function $(l)$ (Yang, 1997) for evolutionary models, and then used likelihood-ratio tests (LRT, $2^{*}\left(l_{2^{-}}\right.$ $\left.l_{1}\right)$ ) to compare competing, nested models that tested for $(I)$ heterogeneity of selection across TR/1 codons (site-specific models, Yang and Nielsen, 2002; Yang et al., 2000; Yang et al., 2005), (ii) selection specific to the NX-2 branch (branch-specific models, Yang and Nielsen, 2002) and (iii) selection at specific codons in the NX-2 branch (branch-site models, Yang and Nielsen, 2002; Zhang et al., 2005). Alternative models were considered significant when LRT statistics exceeded the critical $\chi^{2}$ value at $P<$ 0.05 , with degrees of freedom equal to the difference in the number of parameters in the two models (Yang and Nielsen, 2002; Yang et al., 2000; Zhang et al., 2005).

\section{Results}

\subsection{Geographic distribution of NX-2}

Using a PCR-RFLP assay targeting variation in TRI1, we surveyed 2,515 F. graminearum isolates from 19 different countries to assess the geographic distribution of the NX-2 TRI1 allele. Isolates with the NX-2 allele were limited to southern Canada and the northern U.S., where they were observed at low frequency (1.8\%). Only $2.2 \%$ of F. graminearum isolates from the Upper Midwest of the U.S. and south central and 
southeastern Canada had the NX-2 TRI1 allele, and in Connecticut (northeastern U.S.) only $1.1 \%$ had the NX-2 allele (Fig. 2). Six of the NX-2 isolates were recovered from cereal crops grown within the previously documented range of NX-2 isolates: one isolate from wheat in Minnesota (north central U.S.) and five isolates from barley in Québec (southeastern Canada). Two other NX-2 isolates were recovered from corn stubble in Connecticut, U.S., and another isolate was recovered from oats in Manitoba (southwestern Canada). All nine isolates identified as NX-2 with the PCR-RFLP assay had the 3ADON TRI-cluster MLGT genotype. The NX-2 allele was absent from the other regions of the U.S. that were sampled, which included North Carolina (eastern U.S., $N=$ 219), Pennsylvania (northeastern U.S., $N=171$ ), Indiana/Kentucky (midwestern U.S., $N$ $=51$ ), and Louisiana (southern U.S., $N=99$ ). In addition, none of the 1,471 F. graminearum isolates sampled from Europe, Asia, Africa, South America and Australia had the NX-2 TRI1 allele.

\subsection{Phylogenetic Analyses}

A concatenated six-gene phylogeny (Fig. 3) resolved NX-2 isolates and type B F. graminearum in a monophyletic clade with $100 \%$ bootstrap support, confirming that NX2 isolates were nested within a monophyletic $F$. graminearum. The maximum likelihoodinferred TRI1 phylogeny (Fig. 4) strongly supported a monophyletic FSAMSC-1 clade, but was incongruent with the evolutionary history of species divergence within FSAMSC-1. For example, F. graminearum was polyphyletic in the $T R / 1$ tree, and $F$. graminearum isolates with the NX-2 allele were recovered in 99\% of bootstrap replicates as a highly divergent sister-clade to all other members of FSAMSC-1. A phylogeny inferred from Tri1 amino acids (data not shown) was highly similar in 
topology to the $T R / 1$ tree and also showed strong evidence (100\% bootstrap support) for divergence of NX-2 F. graminearum from the other FSAMSC-1 isolates.

Consequently, a constraint tree specifying $F$. graminearum monophyly provided a significantly worse fit to the data than the unconstrained TR/1 tree (SH test, $P<0.01$ ). Although the placement of the divergent NX-2 clade was the most obvious conflict between the TR/1 tree and the recognized species phylogeny, additional conflicts were observed. Early diverging heterothallic species, such as $F$. lunulosporum and $F$. culmorum were nested within the FGSC, and SH tests confirmed that the fit of a constraint tree specifying FGSC monophyly was significantly worse than the unconstrained TR/1 phylogeny $(P<0.01)$ even when NX-2 sequences were removed from the analysis.

In order to minimize the influence of natural selection on reconstruction of the TRI1 gene tree, we repeated the phylogenetic analyses for TRI1 using only intron and four-fold degenerate sites (neutral sites). The maximum likelihood phylogeny based on neutral sites (Fig. 5) was consistent with the all-sites tree inferred for TRI1 (Fig. 4) in that neither the FGSC nor $F$. graminearum were recovered as monophyletic groups and NX-2 alleles formed a strongly supported monophyletic clade. However, the NX-2 clade was deeply nested within FSAMSC-1 in the neutral site tree, in contrast to the divergent position of the NX-2 clade in the all-sites tree. In addition, neutral site evolution at TR/1 did not strongly contradict a monophyletic $F$. graminearum $(\mathrm{SH}$ test based on a constraint of $F$. graminearum monophyly, $P>0.05$ ). However, there was significant ( $\mathrm{SH}$ test, $P<0.05)$ support for a paraphyletic FGSC in the neutral site phylogeny even when 
NX-2 isolates were omitted from the analyses, as was observed when all sites were considered.

\subsection{TR/1 flanking regions}

Given the unexpected relationships observed among FSAMSC-1 isolates in the $T R / 1$ gene tree, we sequenced TRI1 flanking regions to determine if $T R / 1$ was present in the same genomic context, and thus likely to be orthologous, across FSAMSC-1. Comparison of flanking sequences indicated that $T R / 1$ was likely located in the same genomic context (GC1) in all FSAMSC-1 isolates; including isolates with the NX-2 allele (Fig. 6A). Interestingly, gene trees derived from genes flanking TRI1 (FGSG_0070 and FGSG_0072) were also incongruent with the species phylogeny for FSAMSC-1 (Supplementary Fig. 1 and 2). As we observed for TRI1, the FGSC and F. graminearum were non-monophyletic in gene trees for FGSG_00070 or FGSG_00072 (SH tests, $P<$ 0.01). Unlike $T R I 1$ however, NX-2 isolates of $F$. graminearum did not form a monophyletic clade within the maximum likelihood phylogeny for FGSG_00070 or FGSG_00072. Neutral (synonymous) site phylogenies inferred for FGSG_00070 and FGSG_00072 (data not shown) were highly consistent with the all-sites gene trees and also depicted the FGSC and NX-2 F. graminearum as polyphyletic.

\subsection{Molecular evolution of NX-2 Tri1 enzymes}

Comparison of predicted Tri1 amino acid sequences from the NX-2 and type B F. graminearum included in the phylogenetic analyses revealed 13 fixed differences between these two groups (Fig. 6B). Sequencing of TR/1 from a geographically diverse panel of 239 additional F. graminearum identified as type B using the PCR-RFLP assay confirmed this observation (Supplementary Table 1). In addition, the NX-2 character 
states at these 13 amino acid sites were not observed among other FSAMSC-1 species, and only four $(33 \mathrm{~T}, 100 \mathrm{~N}, 210 \mathrm{~T} 256 \mathrm{~N})$ were shared with any of the TR/1 alleles sampled from outside FSAMSC-1. The accumulation of nonsynonymous substitutions specific to the NX-2 clade indicated that the evolution of NX-2 may have been accompanied by changes in selection pressure acting on Tri1.

We used likelihood ratio tests (LRTs) and parameter estimates derived from different codon models of molecular evolution (Table 1 ) to test for $(I)$ heterogeneity of selective constraint among TRI1 codons, (ii) sites within TR/1 that evolved by positive selection, and (iii) changes in selective constraint along the branch leading to the NX-2 clade (NX-2 branch). Site-specific models indicated heterogeneity of $\omega$ across TRI1 codons (M0 versus M3), although tests for site-specific positive selection (M1a versus M2a, M7 versus M8) were not significant across the TR/1 gene tree as a whole (Fig. 4). A branch-model allowing for a different $\omega$ along the NX-2 branch $\left(\mathrm{M}_{\mathrm{NX}-2}\right)$ fit the data significantly $(P<0.001)$ better than a model assuming a single $\omega$ for all branches in the TRI1 gene tree (M0), indicating that the evolution of NX-2 was accompanied by a significant change in selective pressure on TR/1. The estimate of $\omega$ along the NX-2 branch was more than six-fold higher than for background branches (Table 1) and was consistent with positive selection $(\omega=1.16)$. However, a direct test of positive selection along the NX-2 branch was not significant, as the $\mathrm{M}_{\mathrm{NX}-2}$ model failed to provide a significant improvement over a branch model in which the NX-2 branch evolved neutrally ( $\mathrm{MO}_{\mathrm{NX}-2}$ neutral). Branch-site models allowing for variation of $\omega$ among $T R / 1$ codons and a change in $\omega$ associated with NX-2 TRI1 (Model A) indicated that $9 \%$ of codons had evolved by positive selection along the NX-2 branch, but again direct tests 
of site-specific positive selection along the NX-2 branch (Model A versus Model A neutral) were not significant $(P=0.11)$.

\section{Discussion}

Our analyses of $T R / 1$ variation within a global collection of 2,515 F. graminearum isolates indicated that the NX-2 trichothecene type could be indigenous, and possibly endemic, to parts of both the northern U.S. and southern Canada. Previous analyses of the distribution and prevalence of NX-2 isolates were restricted to Canada and the Upper Midwest of the U.S., where the NX-2 type was observed among $2.8 \%$ and $1.7 \%$ of wheat isolates respectively (Kelly et al., 2015; Liang et al., 2014). We found a similar frequency of NX-2 isolates in those areas (2.2\%), and the current results expanded the known geographic distribution of NX-2 strains to include the northeastern U.S. (Connecticut, 1.1\%). However, the NX-2 allele was not observed among $540 F$. graminearum isolates sampled from other regions of the U.S. and 1,471 isolates sampled from Europe, Asia, Africa, South America and Australia. Although the NX-2 allele likely exists outside the expanded geographic range identified in this study, and may be locally prevalent is some regions, the current data indicate that NX-2 is not common across the cereal production regions sampled.

A total of $41 \mathrm{NX}-2 \mathrm{~F}$. graminearum isolates have now been identified, and as in previous publications (Kelly et al., 2015; Liang et al., 2014; Varga et al., 2015), the NX-2 isolates identified in this study have 3ADON TRI-cluster types. In Canada and the northern U.S., the 3ADON TRI-cluster type is strongly associated with the emergence and spread of the NA2 genetic population of $F$. graminearum, which has a range that is similar to that of the NX-2 chemotype (Gale et al., 2007; Schmale et al., 2011; Ward et 
al., 2008). Despite extensive sympatry NX-2 isolates have a genetic background that distinguishes them from the emergent NA2 (predominantly 3ADON) genetic population and the putatively native NA1 (predominantly $15 \mathrm{ADON}$ ) genetic population that is dominant across much of the U.S. and Canada (Kelly et al., 2015; Liang et al., 2014). Although this apparent lack of gene flow could be expected if NX-2 strains were only recently introduced into this region, we were unable to identify the NX-2 allele in the other geographic and genetic populations sampled, including those from Louisiana, Norway, Finland, Russia, and Japan that have appreciable frequencies of strains with 3ADON TRI-cluster types (Aamot et al., 2015; Gale et al., 2011; Suga et al., 2008; YliMattila et al., 2009). Likewise, phylogenetic analyses of a multi-gene dataset indicated that NX-2 isolates were not closely related to the divergent Gulf Coast population of $F$. graminearum (Fig. 3), which also includes strains with the 3ADON TRI-cluster type (Gale et al., 2011; Starkey et al., 2007). Genetic integration could also be limited by host-mediated barriers to reproduction or other forms of differential niche adaptation. Although NX-2 isolates have been recovered from cereal crops that are commonly infected with other $F$. graminearum, the first NX-2 strain described was recovered from non-agricultural grasses, indicating a potential host reservoir separate from commercial cereals (Varga et al., 2015).

Analyses of $T R I 1$ variation also expanded the known host range of NX-2 strains, which accounted for $6.0 \%$ of isolates from barley in Québec and 3.3\% of oat isolates from Manitoba (Supplementary Table 1). All of the NX-2 isolates identified in previous surveys of cereal crops were obtained from wheat, and the absence of the NX-2 type among 247 F. graminearum isolates from barley in the Upper Midwest led Liang et al., 
(2015) to question if NX-2 isolates were adapted to wheat, but not barley. Our data indicated NX-2 strains are capable of infecting barley; however, regional differences in NX-2 prevalence on specific hosts may exist. Consistent with findings reported by Liang et al. (2015), NX-2 was not observed among the 54 barley isolates we examined from Manitoba (Supplementary Table 1). Previous analyses indicated that a complex adaptive landscape has driven regional differences in the prevalence of trichothecene chemotypes (3ADON and 15ADON) and genetic populations (NA1 and NA2) that distinguish eastern Canada from the Canadian prairie-provinces and the Upper Midwest (Kelly et al., 2015), and similar regional differences were reported for $F$. asiaticum in China (Yang et al., 2008; Zhang et al., 2012). It is possible that regional differences could also influence FHB pathogen composition in relation to specific hosts, but additional surveys and direct experimental analyses of pathogen aggressiveness and host susceptibility will be required to address this question.

We conducted phylogenetic analyses of $T R / 1$ sequences in order to understand the evolutionary origins of the NX-2 chemotype. This effort was complicated by the fact that relationships observed across the TR/1 gene tree (Fig. 4 and Fig. 5) were incongruent with the species phylogeny (Fig. 3, Aoki et al., 2015; Sarver et al., 2011). The results were indicative of trans-species evolution, in which ancestral polymorphism at TR/1 persisted through multiple speciation events within FSAMSC-1 and was either maintained or subsequently sorted into descendent lineages in a way that does not reflect phylogenetic relationships of species within FSAMSC-1. These findings are consistent with previous evidence of trans-species evolution of TR/1 alleles (Proctor et al., 2009), but provide the first documentation that this phenomenon extended to $T R / 1$ 
evolution within FSAMSC-1. Trans-species evolution was also previously observed for TRI-cluster genes within FSAMSC-1, where trans-species polymorphism was clearly associated with the maintenance of trichothecene chemotype diversity by natural selection (Ward et al., 2002). Proctor et al. (2009) suggested trans-species evolution at TRI1 might also be related to the production of structurally diverse trichothecenes. However, this does not appear to be the case within FSAMSC-1, as patterns of transspecies evolution were evident among the type B trichothecene-producing strains, all of which produce 8-keto, 7-hydroxy trichothecenes (Fig. 4 and Fig. 5). We also observed patterns of phylogenetic incongruence consistent with trans-species evolution at genes flanking TRI1 (FGSG_0070 and FGSG_0072) within the genomic context shared by all members of FSAMSC-1. In this genomic context (GC1), TRI1 is positioned near the telomere of chromosome 1 in F. graminearum, in a region that is SNP-dense and enriched for genes that encode secreted effector proteins predicted to be involved in fungus-plant interactions (Cuomo et al., 2007; Zhao et al., 2014). As such, patterns of trans-species evolution at TRI1 may be an indirect result of evolutionary pressures acting on a linked locus that is not involved in trichothecene biosynthesis.

Initial phylogenetic analyses based on all sites within TRI1 suggested that NX-2 evolved early in the history of FSAMSC-1 (Fig. 4). However, this appeared to be an artifact owing to the accumulation of nonsynonymous mutations in the TR/1 gene of NX2 isolates (Fig. 6). When we corrected for the influence of selection, analyses of intron sequences and four-fold degenerate sites demonstrated that NX-2 evolved more recently from a type B TR/1 allele (Fig. 5), possibly following diversification of $F$. graminearum (non-significant SH test of optimal tree versus a constraint specifying $F$. 
graminearum monophyly). By testing the likelihood of different codon models of molecular evolution, we demonstrated that NX-2 evolution was associated with a significant change in selection pressure operating on Tri1. Therefore, the extreme divergence of NX-2 isolates in the TR/1 gene tree appears to be the result of an evolutionary process distinct from that responsible for trans-species evolution of $T R / 1$ polymorphism. Parameter estimates from codon models were consistent with positive selection along the NX-2 branch of the TRI1 gene tree (Table 1), and were indicative of adaptive evolution at specific amino-acid sites. However, direct tests of positive selection versus effectively neutral evolution were not significant. Varga et al. (2015) hypothesized that the NX-2 toxin could provide an advantage to the fungus by circumventing glutathione-mediated detoxification in-planta, and our analyses provide indications that specific mutations associated with the evolution of NX-2 may have been favored by natural selection. However, given the lack of statistical significance in support of positive selection we can conclude only that NX-2 producers evolved in an environment in which the loss of 8-keto trichothecenes was not opposed by purifying selection. Either way, the observation of chemotype-specific differences in selective constraint on Tri1 evolution further indicated that NX-2 strains occupy an evolutionary niche that is distinct from type B strains of $F$. graminearum.

The predicted amino acid sequences for Tri1 among NX-2 isolates were distinguished from those of all other FSAMSC-1 isolates by 13 differences (Fig. 6B). Varga et al. (2015) previously reported 14 predicted amino acid differences between NX-2 and type B isolates of $F$. graminearum, however the NX-2 character state at one of these sites (363) is shared with an isolate from the Gulf Coast population of $F$. 
graminearum as well as strains from at least nine other FGSC species that produce type B trichothecenes. In comparing $T R / 1$ sequences with those from a broader group of Fusarium, we determined that only nine of these predicted amino acid differences were unique to NX-2 isolates. These included the I361F mutation located within one of six substrate recognition sites (Gotoh, 1992; Marchler-Bauer et al., 2015). Mutations at this site can drastically change P450 substrate specificity (Lindberg and Negishi, 1989), and substitutions in proximity to residue 361 have been shown to alter the location of substrate hydroxylation (Schalk and Croteau, 2000). In addition, the A450V mutation, located within the highly conserved heme-binding loop of the enzyme (Fig. 6B), was found exclusively in NX-2 isolates. Mutations in this region can alter heme-substrate affinity and substrate turnover (Warrilow et al., 2010), which could explain the functional difference between NX-2 and other Tri1 enzymes. Site-directed mutagenesis experiments targeting the amino acid residues we identified as specific to NX-2 producers should lead to the identification of mutations responsible for the NX-2 chemotype and to the further development of molecular methods for the specific detection of chemotype-determining mutations as part of pathogen monitoring efforts.

\section{Conclusions}

We conducted a molecular survey of $T R / 1$ to improve understanding of the ecological and toxicological significance of the recently identified NX-2 chemotype by providing the first data on the distribution and prevalence of NX-2 strains from a global collection of $F$. graminearum isolates. The results of this study expanded the known geographic distribution of NX-2 strains to include the northeastern U.S., expanded the known host range of NX-2 strains to include oat and barley, and indicated that the NX-2 
chemotype may be indigenous, and possibly endemic, to parts of the northern U.S. and southern Canada. Phylogenetic and molecular evolution analyses provided evidence that TR/1 variation within FSAMSC-1 was shaped by trans-species evolution of ancestral polymorphism, and indicated that the NX-2 chemotype evolved relatively recently from a type $B$ ancestor as a result of a significant change in selective pressures acting on Tri1. Our results indicate contamination of cereals with NX-2 or its derivatives is likely to be geographically limited in comparison to type B trichothecenes. However, evidence that the NX-2 chemotype evolved in response to a significant change in selective pressure suggests the need for additional monitoring of this novel type A trichothecene across a broad range of hosts, not only to identify potential source populations, but also to track potential range expansions.

\section{Acknowledgements}

We thank N. Orwig and T. Usgaard for excellent technical support. We also are indebted to Kerry O'Donnell, Sukumar Chakraborty, Emerson Del Ponte, H. Corby Kistler, David Geiser, the Pennsylvania State University Fusarium Research Center, and the ARS Culture Collection (NRRL) for assistance in developing the isolate collections used in this study. This study was supported by the United States Department of Agriculture-Agricultural Research Service National Program for Food Safety. Mention of trade names or commercial products in this article is solely for the purpose of providing specific information and does not imply recommendation or endorsement by the United States Department of Agriculture (USDA). USDA is an equal opportunity provider and employer. 
Table 1. Parameter estimates for codon models of molecular evolution.

\begin{tabular}{|c|c|c|}
\hline Site Models ${ }^{1}$ & Likelihood & Parameter Estimates $^{2}$ \\
\hline M0 (single $\omega$ ) & -11704.18 & $\omega=0.17$ \\
\hline M1a (neutral) & -11583.19 & $p_{0}=0.87, \omega_{0}=0.11 ; p_{1}=0.13, \omega_{1}=1.0$ \\
\hline M2a (selection) & -11583.19 & $\begin{array}{c}p_{0}=0.87, \omega_{0}=0.11 ; p_{1}=0.13, \omega_{1}=1.0 ; p_{2}=0.00 \\
\omega_{2}=27.69\end{array}$ \\
\hline M3 (multiple $\omega$ ) & -11527.53 & $\begin{aligned} p_{0}=0.40, \omega_{0}=0.01 ; p_{1} & =0.48, \omega_{1}=0.2 ; p_{2}=0.12 \\
\omega_{2} & =0.69\end{aligned}$ \\
\hline M7 (neutral) & -11529.97 & $p=0.47, q=2.07$ \\
\hline M8 (selection) & -11529.45 & $p=0.52, q=2.58 ; p_{1}=0.02, \omega_{1}=1.0$ \\
\hline \multicolumn{3}{|l|}{ Branch Models } \\
\hline $\mathrm{M} 0_{\mathrm{NX}-2}$ & -11696.80 & $\omega_{0}=0.17 ; N X-2 \omega=1.16$ \\
\hline $\begin{array}{c}M 0_{N X-2} \text { neutral } \\
(\text { fix NX-2 } \omega=1)\end{array}$ & -11696.84 & $\omega_{0}=0.17 ; N X-2 \omega=1.0$ \\
\hline \multicolumn{3}{|c|}{ Branch-Site Models } \\
\hline $\begin{array}{l}\text { Model A neutral } \\
\qquad\left(\text { fix } \omega_{2}=1\right)\end{array}$ & -11576.65 & $\begin{array}{l}p_{0}=0.26, \text { Background } \omega_{0}=0.11, N X-2 \omega_{0}=0.11 ; \\
p_{1}=0.04, \text { Background } \omega_{1}=1.0, N X-2 \omega_{1}=1.0 ; \\
p_{2}=0.61, \text { Background } \omega_{0}=0.11, N X-2 \omega_{2}=1.0 ; \\
p_{3}=0.09, \text { Background } \omega_{1}=1.0, N X-2 \omega_{2}=1.0 ;\end{array}$ \\
\hline Model A & -11575.36 & $\begin{array}{l}p_{0}=0.79, \text { Background } \omega_{0}=0.11, N X-2 \omega_{0}=0.11 ; \\
p_{1}=0.12, \text { Background } \omega_{1}=1.0, N X-2 \omega_{1}=1.0 ; \\
p_{2}=0.08, \text { Background } \omega_{0}=0.11, N X-2 \omega_{2}=11.10 ; \\
p_{3}=0.01, \text { Background } \omega_{1}=1.0, N X-2 \omega_{2}=11.10 ;\end{array}$ \\
\hline
\end{tabular}

${ }^{1}$ Site models described in Yang et al., (2005); branch models described in Yang and Nielsen (2002); branch-site models described in Zhang et al., (2005).

${ }^{2} \omega$ is the ratio of non-synonymous to synonymous substitution rates; $p$ is the proportion of sites at TRI1 with associated $\omega$. For models M7 and M8 $p$ and $q$ represent the shape parameter estimates for the $\beta$ distribution (Yang et al., 2000). 
Fig. 1. Chemical structures of 3-acetyl deoxynivalenol, a 7-hydroxy, 8-keto, type $B$ trichothecene; NX-2, a 7-hydroxy type A trichothecene; and T-2 toxin, a 8-acyl type A trichothecene.

Fig. 2. Geographic sampling of 2,515 F. graminearum isolates included in the PCRRFLP survey of the NX-2 TRI1 allele. N indicates the number of isolates screened from each sampled country (shaded grey). Delineated range in Canada and the U.S. is based on the locations of isolates that were identified as NX-2 based on PCR-RFLP and TRl1 gene sequences.

Fig. 3. Maximum likelihood phylogeny based on concatenated DNA alignment of six genes previously used to infer phylogenetic relationships in FSAMSC-1: $\beta$-tubulin (TUB2), translation elongation factor $1 \alpha$ (TEF1), trichothecene-3-O-acetyltransferase (TR/101), phosphate permease (PHO5), histone H3 (HIS3) and reductase (RED). The tree was inferred using the Tamura 3-parameter model of nucleotide substitution (Tamura, 1992) with a Gamma parameter to account for rate heterogeneity. Bootstrap values (\%, based on 1000 replications) $\geq 70$ are shown on branches. The tree was rooted at midpoint and drawn to scale, with branch lengths measured in the number of substitutions per site.

Fig. 4. Maximum likelihood phylogeny of $T R / 1$ gene sequences $(N=77$ after duplicate sequences from the same species were removed) from type $A$ and type $B$ trichothecene-producing isolates of Fusarium. The phylogeny was inferred using the Kimura 2-parameter model of nucleotide substitution (Kimura, 1980) with a Gamma parameter to account for rate heterogeneity. Bootstrap values (\%, based on 1000 replications) $\geq 70$ are indicated on branches. The tree was rooted at midpoint and drawn to scale, with branch lengths measured in the number of substitutions per site. Trichothecene toxin chemotype is indicated in parentheses for FSAMSC-1 isolates.

Fig. 5. Phylogeny based on nucleotide sequences of introns and four-fold degenerate sites of $T R / 1$ from type A and type B trichothecene-producing isolates of Fusarium ( $N=$ 77). The tree was inferred using the Tamura 3-parameter model of nucleotide substitution (Tamura, 1992), with a Gamma parameter to account for rate heterogeneity and a proportion of invariable sites. The tree was rooted at midpoint and drawn to scale, with branch lengths measured in the number of pairwise differences per site. The tree to the left is an enlargement of the FSAMSC-1 clade in the smaller tree to the right. Bootstrap values (\%, based on 1000 replications) $\geq 70$ are indicated on branches.

Fig. 6. A, Genomic context GC1 of TRI1 in NX-2 isolates and other members of the $F$. sambucinum species complex (FSAMSC-1). Gene designations and chromosomal coordinates are as described for F. graminearum strain $\mathrm{PH}-1$ in the Fusarium Comparative Database (Broad Institute, Accession GCA_000240135.3). The TR/16 $\Psi$ region has a nonfunctional version of the trichothecene acyl transferase gene, TR/16 (Brown et al., 2003; McCormick et al., 2004; Proctor et al., 2009). B, Locations of introns, exons, functional domains and nonsynonymous mutations in TR/1/Tri1 of NX-2 F. graminearum. TRI1 exons correspond to the black or grey regions; the grey regions 
indicate conserved cytochrome P450 monooxygenase domain (amino acids 291-468, Marchler-Bauer and Bryant, 2004; Marchler-Bauer et al., 2015)), including the 20-amino acid heme-binding loop. * indicates NX-2 Tri1 character state was shared with TRI1 alleles from isolates outside of FSAMSC-1.

Supplementary Fig. 1. Maximum likelihood phylogeny inferred from coding sequences of FGSG_00070 from type A and type B trichothecene-producing isolates of Fusarium $(N=64)$ using the Hasegawa-Kishino-Yano model of nucleotide substitution (Hasegawa et al., 1985) with a Gamma parameter to account for rate heterogeneity and a proportion of invariable sites. NX-2 isolates are indicated by arrows. Bootstrap values (\%, based on 1000 replications) $\geq 70$ are indicated on branches. The tree was rooted at midpoint and drawn to scale, with branch lengths measured in the number of substitutions per site.

Supplementary Fig. 2. Maximum likelihood phylogeny inferred from coding sequences of FGSG_00072 from type A and type B trichothecene-producing isolates of Fusarium $(N=63)$ using the Kimura 2-parameter model of nucleotide substitution (Kimura, 1980) with a Gamma parameter to account for rate heterogeneity. NX-2 isolates are indicated by arrows. Bootstrap values (\%, based on 1000 replications) $\geq 70$ are indicated on branches. The tree was rooted at midpoint and drawn to scale, with branch lengths measured in the number of substitutions per site. 


\section{References}

Aamot, H., et al., 2015. Genetic and phenotypic diversity within the Fusarium graminearum species complex in Norway. European Journal of Plant Pathology. $1-19$.

Alexander, N. J., et al., 2009. Genes, gene clusters, and biosynthesis of trichothecenes and fumonisins in Fusarium. Toxin Reviews. 28, 198-215.

Aoki, T., et al., 2015. Fusarium dactylidis sp. nov., a novel nivalenol toxin-producing species sister to $F$. pseudograminearum isolated from orchard grass (Dactylis glomerata) in Oregon and New Zealand. Mycologia. 107, 409-418.

Bec, S., et al., 2015. Characterization of Fusarium strains recovered from wheat with symptoms of head blight in Kentucky. Plant Disease. 99, 1622-1632.

Boutigny, A.-L., et al., 2014. Diversity of the Fusarium graminearum species complex on French cereals. European Journal of Plant Pathology. 138, 133-148.

Boutigny, A.-L., et al., 2011. Analysis of the Fusarium graminearum species complex from wheat, barley and maize in South Africa provides evidence of speciesspecific differences in host preference. Fungal Genetics and Biology. 48, 914920.

Brown, D. W., et al., 2003. Characterization of a Fusarium 2-gene cluster involved in trichothecene C-8 modification. Journal of Agricultural and Food Chemistry. 51, 7936-7944.

Cuomo, C. A., et al., 2007. The Fusarium graminearum genome reveals a link between localized polymorphism and pathogen specialization. Science. 317, 1400-1402.

Del Ponte, E. M., et al., 2015. Regional and field-specific factors affect the composition of Fusarium head blight pathogens in subtropical no-till wheat agroecosystem of Brazil. Phytopathology. 105, 246-254.

Desjardins, A. E., et al., 1996. Reduced virulence of trichothecene-nonproducing mutants of Gibberella zeae in wheat field tests. MPMI-Molecular Plant Microbe Interactions. 9, 775-781.

Edgar, R. C., 2004. MUSCLE: multiple sequence alignment with high accuracy and high throughput. Nucleic Acids Research. 32, 1792-1797.

Gale, L., et al., 2007. Population subdivision of Fusarium graminearum sensu stricto in the upper Midwestern United States. Phytopathology. 97, 1434-1439.

Gale, L. R., et al., 2011. Nivalenol-type populations of Fusarium graminearum and $F$. asiaticum are prevalent on wheat in southern Louisiana. Phytopathology. 101, 124-134.

Gene Codes Corporation, Sequencher® version 4.10.1 sequence analysis software. Ann Arbor, MI USA, 2010.

Goswami, R. S., Kistler, H. C., 2004. Heading for disaster: Fusarium graminearum on cereal crops. Molecular Plant Pathology. 5, 515-525.

Gotoh, O., 1992. Substrate recognition sites in cytochrome P450 family 2 (CYP2) proteins inferred from comparative analyses of amino acid and coding nucleotide sequences. Journal of Biological Chemistry. 267, 83-90.

Hasegawa, M., et al., 1985. Dating of the human-ape splitting by a molecular clock of mitochondrial DNA. Journal of Molecular Evolution. 22, 160-174.

Jansen, C., et al., 2005. Infection patterns in barley and wheat spikes inoculated with wild-type and trichodiene synthase gene disrupted Fusarium graminearum. 
Proceedings of the National Academy of Sciences of the United States of America. 102, 16892-16897.

Kelly, A. C., et al., 2015. Diversity of Fusarium head blight populations and trichothecene toxin types reveals regional differences in pathogen composition and temporal dynamics. Fungal Genetics and Biology. 82, 22-31.

Kimura, M., 1980. A simple method for estimating evolutionary rates of base substitutions through comparative studies of nucleotide sequences. Journal of Molecular Evolution. 16, 111-120.

Kuhnem, P. R., et al., 2015. Trichothecene genotype composition of Fusarium graminearum not differentiated among isolates from maize stubble, Maize ears, wheat spikes, and the atmosphere in New York. Phytopathology. 695-699.

Lee, T., et al., 2002. Tri13 and Tri7 determine deoxynivalenol-and nivalenol-producing chemotypes of Gibberella zeae. Applied and Environmental Microbiology. 68, 2148-2154.

Liang, J., et al., 2015. Population subdivision of Fusarium graminearum from barley and wheat in the Upper Midwestern United States at the turn of the century. Phytopathology. 105, 1466-1474.

Liang, J., et al., 2014. Temporal dynamics and population genetic structure of Fusarium graminearum in the upper Midwestern United States. Fungal Genetics and Biology. 73, 83-92.

Lindberg, R. L., Negishi, M., 1989. Alteration of mouse cytochrome P450coh substrate specificity by mutation of a single amino-acid residue. Nature. 339, 632-634.

Marchler-Bauer, A., Bryant, S. H., 2004. CD-Search: protein domain annotations on the fly. Nucleic Acids Research. 32, W327-31.

Marchler-Bauer, A., et al., 2015. CDD: NCBI's conserved domain database. Nucleic Acids Research. 43, D222-6.

McCormick, S., et al., 2004. Tri1 in Fusarium graminearum encodes a P450 oxygenase. Applied and Environmental Microbiology. 70, 2044-2051.

McCormick, S. P., et al., 2006. Heterologous expression of two trichothecene P450 genes in Fusarium verticillioides. Canadian Journal of Microbiology. 52, 220-226.

McMullen, M., et al., 2012. A unified effort to fight an enemy of wheat and barley: Fusarium head blight. Plant Disease. 96, 1712-1728.

Meek, I. B., et al., 2003. Tri1 encodes the cytochrome P450 monooxygenase for C-8 hydroxylation during trichothecene biosynthesis in Fusarium sporotrichioides and resides upstream of another new Tri gene. Applied and Environmental Microbiology. 69, 1607-1613.

Miller, J. D., et al., 1991. Trichothecene chemotypes of three Fusarium species. Mycologia. 121-130.

Mudge, A. M., et al., 2006. A role for the mycotoxin deoxynivalenol in stem colonisation during crown rot disease of wheat caused by Fusarium graminearum and Fusarium pseudograminearum. Physiological and Molecular Plant Pathology. 69, 73-85.

Nganje, W. E., et al., Economic impacts of Fusarium head blight in wheat and barley: 1993-2001. Agribusiness and Applied Economics Report No. 538. Department of Agribusiness and Applied Economics, North Dakota State University, ND, US, 2004. 
O'Donnell, K., et al., 1998. Molecular systematics and phylogeography of the Gibberella fujikuroi species complex. Mycologia. 465-493.

O'Donnell, K., et al., 2013. Phylogenetic analyses of RPB1 and RPB2 support a middle Cretaceous origin for a clade comprising all agriculturally and medically important fusaria. Fungal Genetics and Biology. 52, 20-31.

Pestka, J., 2010. Toxicological mechanisms and potential health effects of deoxynivalenol and nivalenol. World Mycotoxin Journal. 3, 323-347.

Proctor, R. H., et al., 2009. Evidence that a secondary metabolic biosynthetic gene cluster has grown by gene relocation during evolution of the filamentous fungus Fusarium. Molecular Microbiology. 74, 1128-1142.

QIAGEN Bioinformatics, CLC Genomics Workbench 8.0.1. 2015.

Qu, B., et al., 2008. Geographic distribution and genetic diversity of Fusarium graminearum and $F$. asiaticum on wheat spikes throughout China. Plant Pathology. 57, 15-24.

Reynoso, M. M., et al., Population structure of Fusarium graminearum species complex genotypes and chemotypes in relation to trichothecenes production. In: T. M. Alconada Magliano, S. N. Chulze, (Eds.), Fusarium Head Blight in Latin America. Springer, Dordrecht, Netherlands, 2013, pp. 3-13.

Sarver, B. A., et al., 2011. Novel Fusarium head blight pathogens from Nepal and Louisiana revealed by multilocus genealogical concordance. Fungal Genetics and Biology. 48, 1096-1107.

Schalk, M., Croteau, R., 2000. A single amino acid substitution (F363I) converts the regiochemistry of the spearmint (-)-limonene hydroxylase from a C6-to a C3hydroxylase. Proceedings of the National Academy of Sciences. 97, 1194811953.

Schmale, D., et al., 2011. Trichothecene genotypes of Gibberella zeae from winter wheat fields in the eastern USA. Plant Pathology. 60, 909-917.

Shimodaira, H., Hasegawa, M., 1999. Multiple comparisons of log-likelihoods with applications to phylogenetic inference. Molecular Biology and Evolution. 16, 1114-1116.

Stamatakis, A., 2014. RAxML version 8: a tool for phylogenetic analysis and postanalysis of large phylogenies. Bioinformatics. 30, 1312-1313.

Starkey, D. E., et al., 2007. Global molecular surveillance reveals novel Fusarium head blight species and trichothecene toxin diversity. Fungal Genetics and Biology. 44, 1191-1204.

Suga, $\mathrm{H}$. , et al., 2008. Molecular characterization of the Fusarium graminearum species complex in Japan. Phytopathology. 98, 159-166.

Tamura, K., 1992. Estimation of the number of nucleotide substitutions when there are strong transition-transversion and $\mathrm{G}+\mathrm{C}$-content biases. Molecular Biology and Evolution. 9, 678-687.

Tamura, K., et al., 2013. MEGA6: Molecular Evolutionary Genetics Analysis Version 6.0. Molecular Biology and Evolution. 30, 2725-2729.

Umpiérrez-Failache, M., et al., 2013. Regional differences in species composition and toxigenic potential among Fusarium head blight isolates from Uruguay indicate a risk of nivalenol contamination in new wheat production areas. International Journal of Food Microbiology. 166, 135-140. 
Varga, E., et al., 2015. New tricks of an old enemy: Isolates of Fusarium graminearum produce a type A trichothecene mycotoxin. Environmental Microbiology. 17, 2588-2600.

Ward, T. J., et al., 2002. Ancestral polymorphism and adaptive evolution in the trichothecene mycotoxin gene cluster of phytopathogenic Fusarium. Proceedings of the National Academy of Sciences. 99, 9278-9283.

Ward, T. J., et al., 2008. An adaptive evolutionary shift in Fusarium head blight pathogen populations is driving the rapid spread of more toxigenic Fusarium graminearum in North America. Fungal Genetics and Biology. 45, 473-484.

Warrilow, A. G., et al., 2010. Azole binding properties of Candida albicans sterol 14- $\alpha$ demethylase (CaCYP51). Antimicrobial Agents and Chemotherapy. 54, 42354245.

Wu, F., et al., 2014. Public health impacts of foodborne mycotoxins. Annual Review of Food Science and Technology. 5, 351-372.

Yang, L., et al., 2008. Fusarium populations on Chinese barley show a dramatic gradient in mycotoxin profiles. Phytopathology. 98, 719-727.

Yang, Z., 1997. PAML: a program package for phylogenetic analysis by maximum likelihood. Computer Applications in the Biosciences: CABIOS. 13, 555-556.

Yang, Z., 2007. PAML 4: phylogenetic analysis by maximum likelihood. Molecular Biology and Evolution. 24, 1586-1591.

Yang, Z., Nielsen, R., 2002. Codon-substitution models for detecting molecular adaptation at individual sites along specific lineages. Molecular Biology and Evolution. 19, 908-917.

Yang, Z., et al., 2000. Codon-substitution models for heterogeneous selection pressure at amino acid sites. Genetics. 155, 431-449.

Yang, Z., et al., 2005. Bayes empirical Bayes inference of amino acid sites under positive selection. Molecular Biology and Evolution. 22, 1107-1118.

Yli-Mattila, T., et al., 2009. A novel Asian clade within the Fusarium graminearum species complex includes a newly discovered cereal head blight pathogen from the Russian Far East. Mycologia. 101, 841-852.

Zhang, H., et al., 2012. Population analysis of the Fusarium graminearum species complex from wheat in China show a shift to more aggressive isolates. PloS One. 7, e31722.

Zhang, J., et al., 2005. Evaluation of an improved branch-site likelihood method for detecting positive selection at the molecular level. Molecular Biology and Evolution. 22, 2472-2479.

Zhao, C., et al., 2014. Relocation of genes generates non-conserved chromosomal segments in Fusarium graminearum that show distinct and co-regulated gene expression patterns. BMC Genomics. 15, 191. 


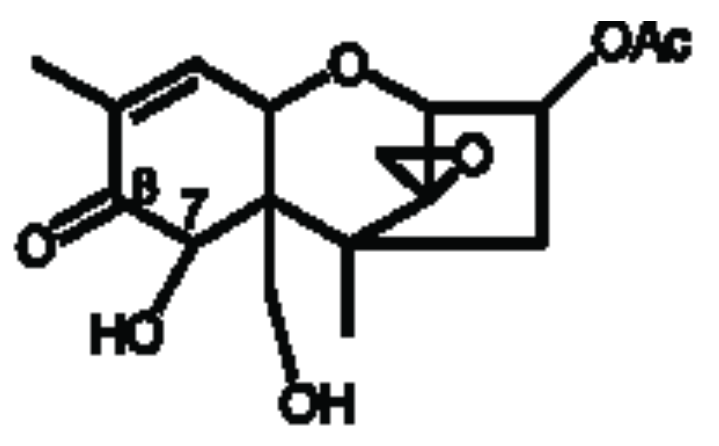

3-acetyl deoxynivalenol (3ADON)

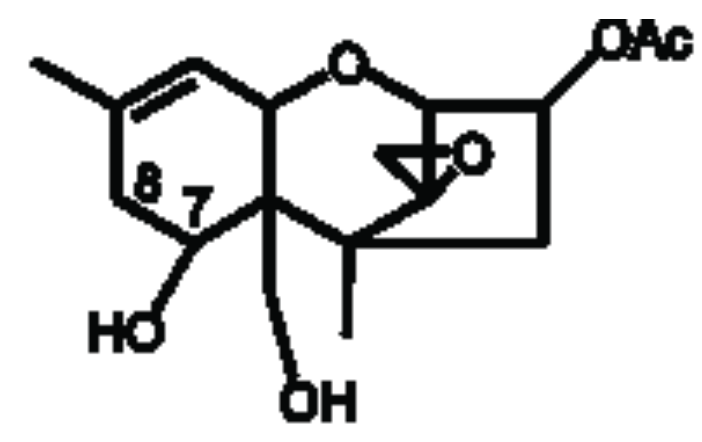

NX-2

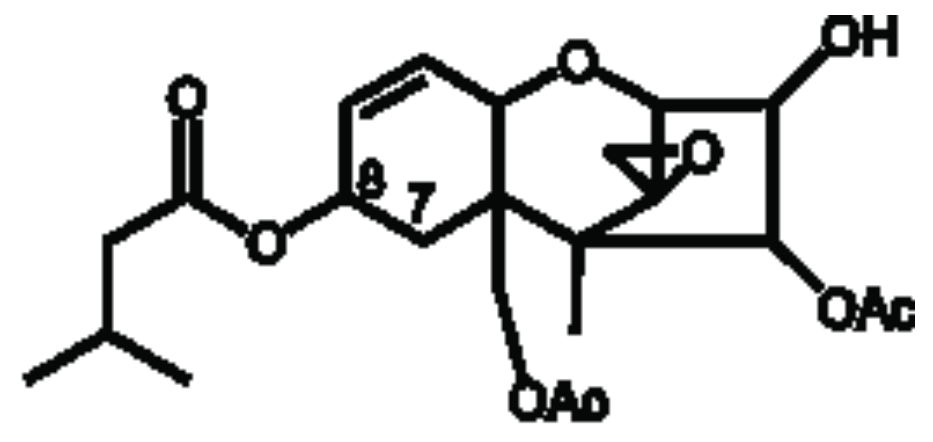

T-2 Toxln 


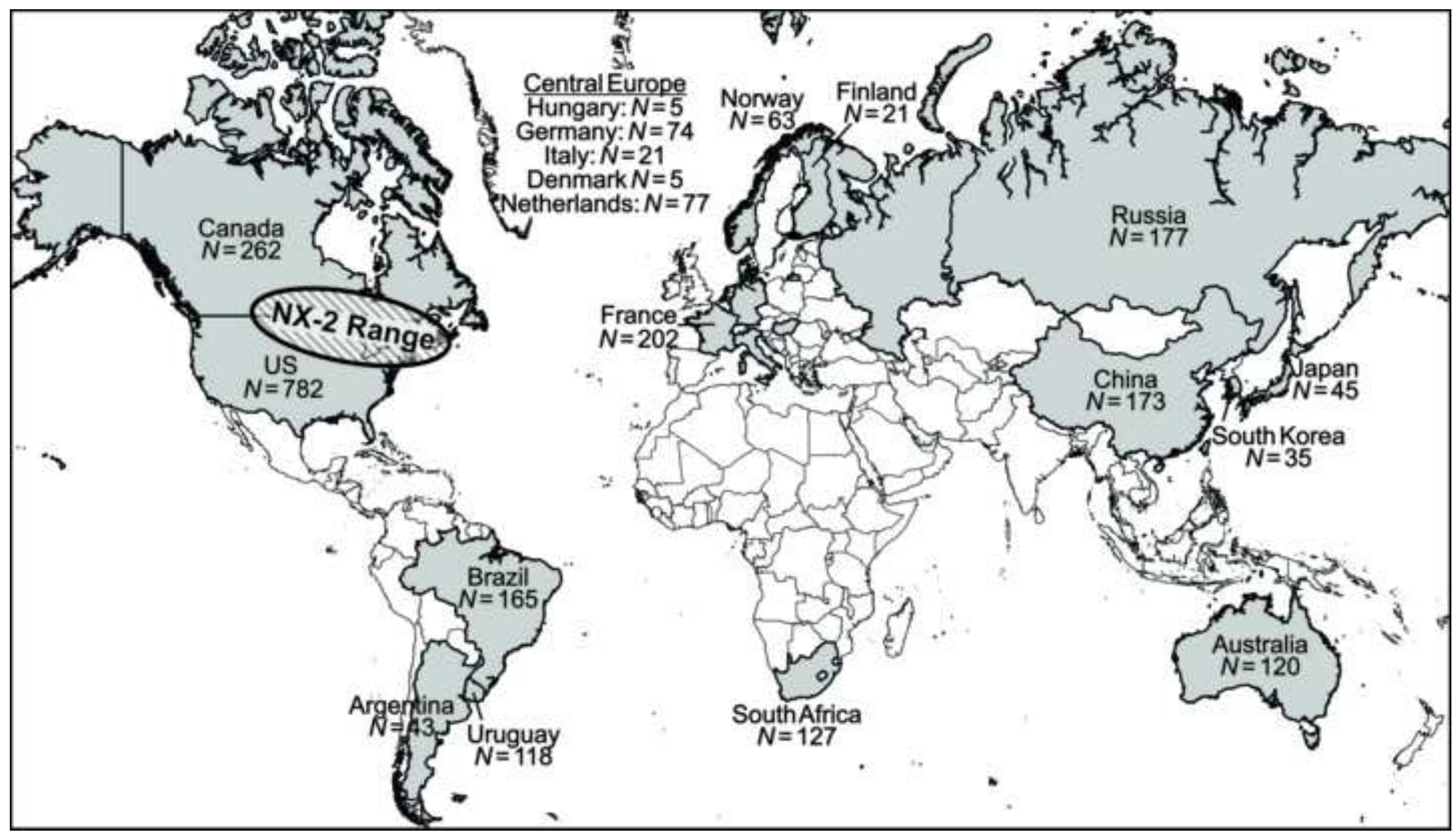




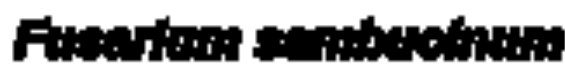

species complex Ilniwes 1 (Fasmso-1)

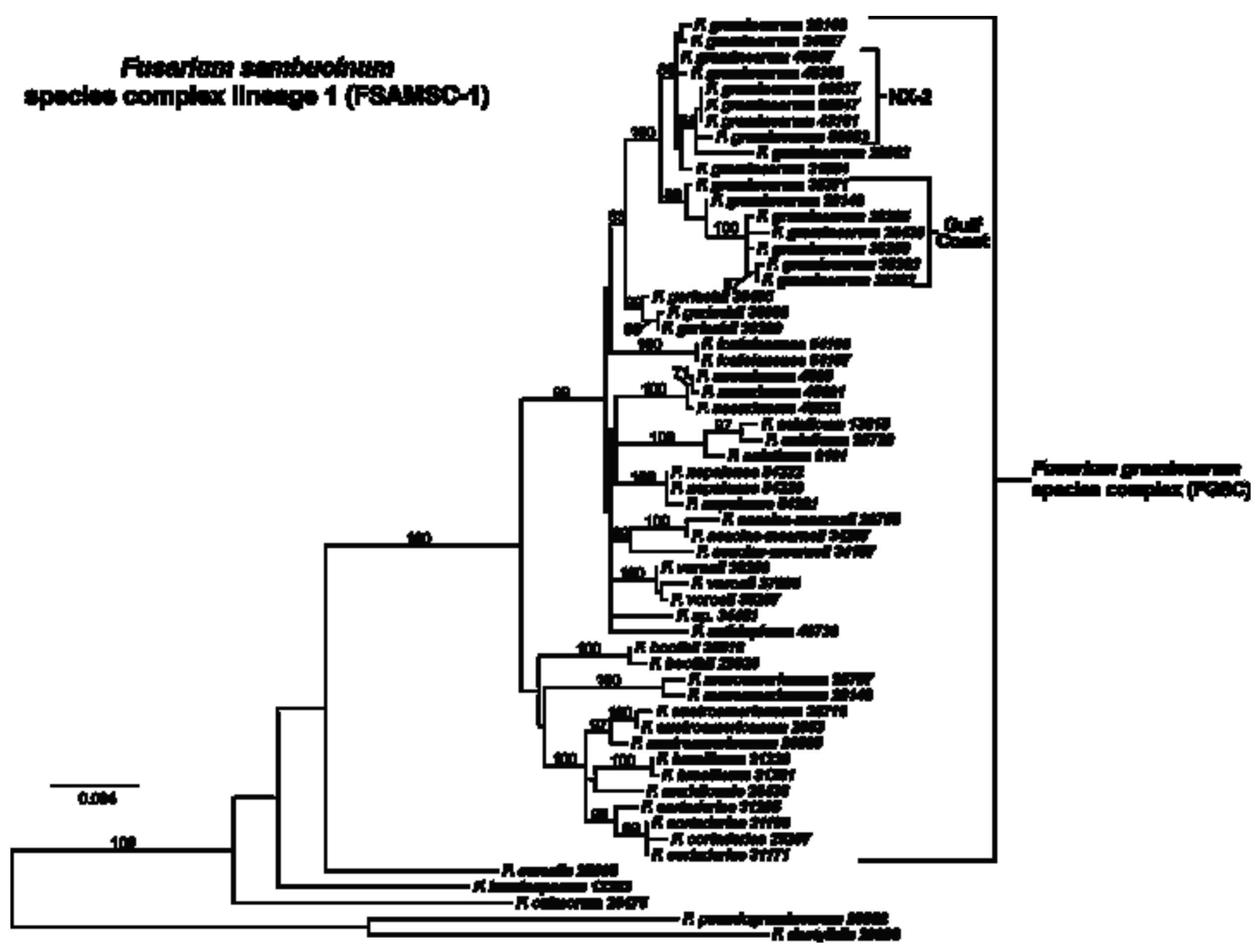




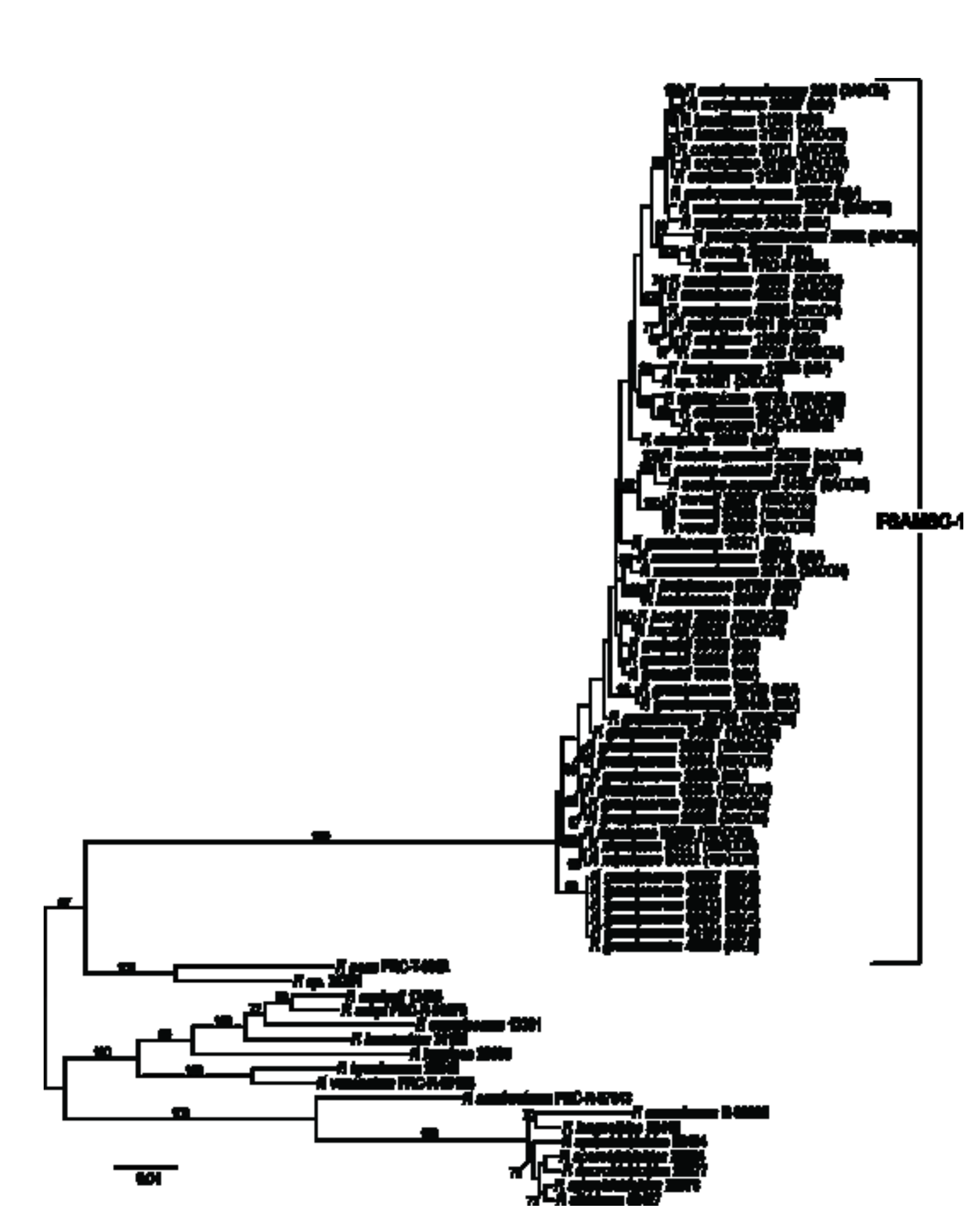

Figure 4

4

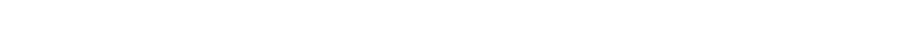

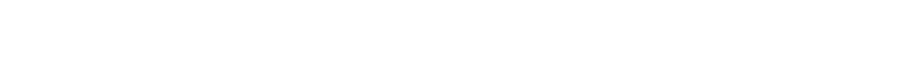

.


Figure 5

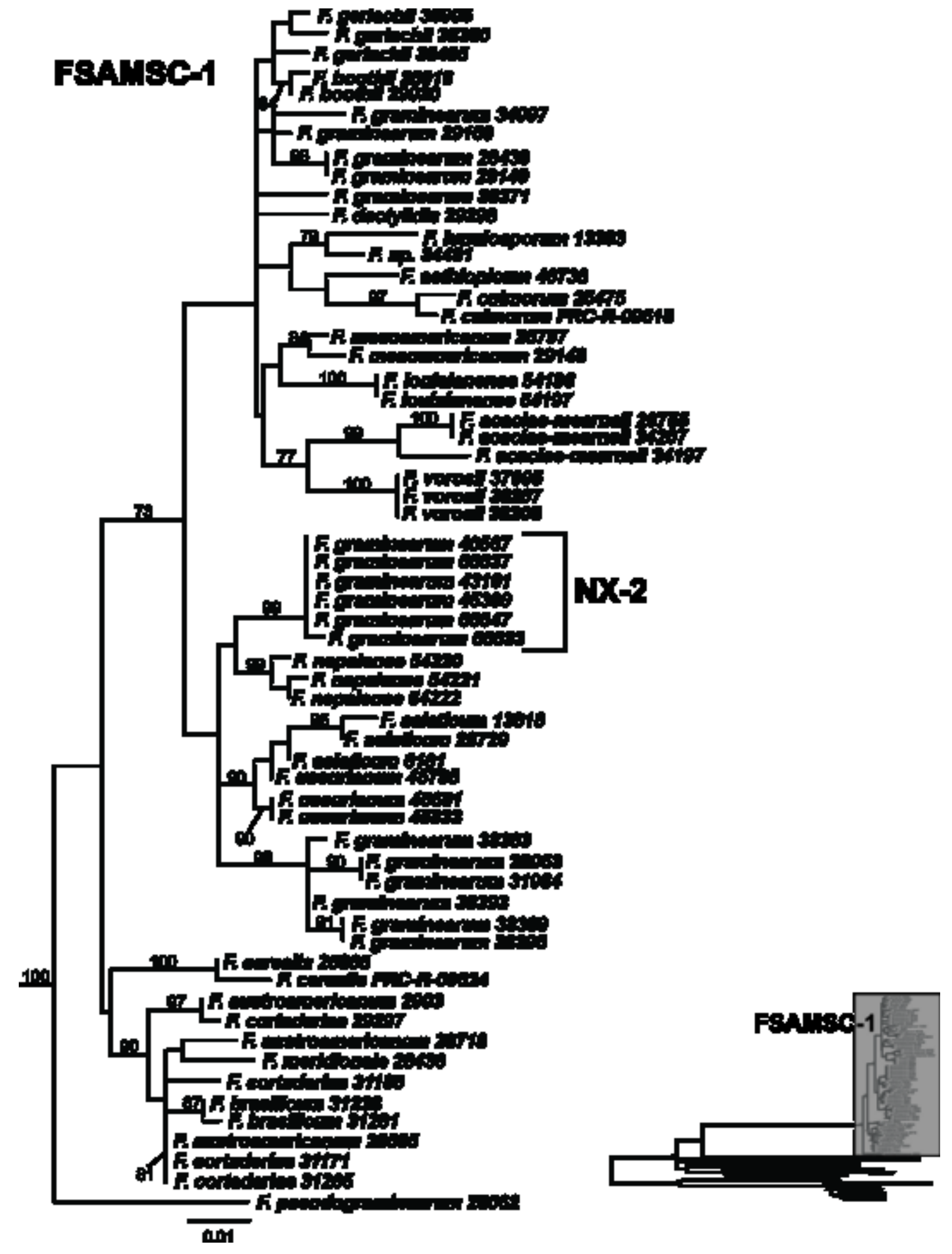




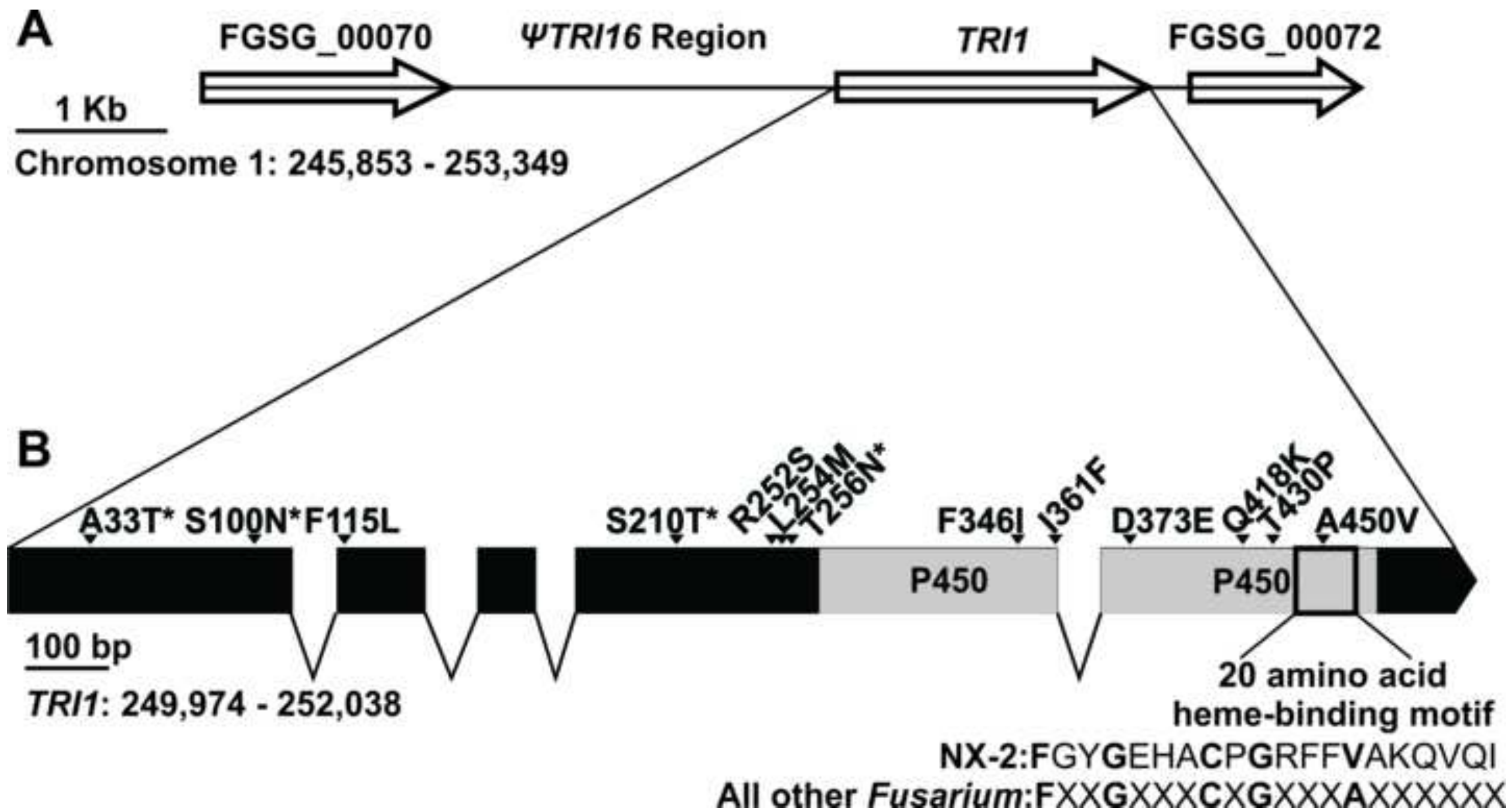

